\title{
Editorial
}

\section{Statistical methods in optimization and operations research}

The current issue is devoted to statistical methods in optimization and operations research (OR). Many interesting and important results have been obtained in recent decades both in OR and Statistics, enriching knowledge and helping to progress in different spheres of human activity. Nevertheless the mutual contribution of OR and statistics can significantly assist in obtaining new results valuable for theory and practice as well. Seven papers contribute to this issue presenting modern statistical methods and their application to various optimization and OR problems, particularly, dynamic and static optimization, continuous and discrete systems with single/multiple-criteria.

The issue is opening with a work by Prof. Igor Mandel, USA, on "Sociosystemics, statistics, decisions" where he introduces principles of a new branch of science, sociosystemics, in conjunction with statistics and decision making. This comprehensive overview could help to transform the social information into a meaningful data available for analysis, prediction, finding innovative solutions, and decision making.

Prof. Galina Dolenko and her colleagues Evgenia Konareva and Dariia Manovytska, Ukraine, in their paper "Fuzzy sets and statistics: application to evaluation of economic development efficiency" evaluate effectiveness of economic development of organizational system by means of the fuzzy sets theory and the elements of applied statistics. The model and the method of evaluation of effectiveness of economic development of organizational system have been proposed. The methodology is illustrated by the statistical data over the years 2009-2010.

Prof. Vitaliy Molostvov, Russia, in his paper "Multiple criteria optimization for stochastic systems with uncertain parameters" considers a "mixed" variant of Multiple Criteria Optimization problem with incomplete information, namely to the case where stochastic distributions for some characteristics of an optimized system are known, but only up to the uncertain parameters. Applied examples are given to show how the combination of stochastic and maximin approaches makes it possible to reduce the problem of this type to multiple criteria optimization problems with uncertainty.

Dr. Soma Roychowdhury together with Dr. Debasis Bhattacharya, India, in their paper "On selection of best system from a group of systems with dependent component lives" present a probabilistic optimization problem of selecting the best system from a group of competing coherent systems with mutually dependent components by optimizing the probability that the system will meet a specified target lifetime and ranking the systems from best to worst according to the orders of those probability values from highest to lowest. The authors select a system by minimizing the probability that the system will fail to meet a specified target lifetime using a stochastic ordering criterion.

Prof. Robert Gogsadze, Prof. Archil Prangishvili, Prof. Rafael Chikovani, Prof. Gela Goderdzishvili, Prof. Alexander Topchishvili, and Prof. Nodar Jibladze from Tbilisi, Georgia, in the paper "Three-dimensional mathematical model of diffusion process during fabrication of isoplanar structure taking into account crystal's anisotropy" develop a mathematical model of a technological process for a semiconductor structure. Such models describe functioning of various devices of micro- and opto-electronics. For practical solving of the boundary problems the authors use methods of tensor analysis, Riemannian geometry, and Green function. Application of these methods allowed determining an analytical image of the impurity concentration depending on time and space variables, topological, physical, and technological parameters of the process and the crystal.

Prof. Rajinder Pal Singh and Prof. Tejwant Singh, India, in the paper "Effect of electrons involved in charge transfer reactions in ECE processes under linear sweep voltametry: a theoretical model" propose a new mathematical model of a mass transport under hydrodynamic conditions at tubular electrodes, in which an irreversible chemical 
reaction is coupled between two reversible charge transfer reactions. They suggest a very interesting way of reducing the boundary value problem into a system of two integral equations, which is solved numerically. The effect of electrons involved in the two charge transfer reactions on voltammograms are investigated and shown graphically.

And the paper "Parameter computation and optimization for phase transitions of the first kind in chemically active materials" by Dr. Boris Vilge, Israel, presents an innovative method for detection of phase transition moments in order to achieve optimum composition of materials and optimum control of their strengthening and hardening processes. Statistical analysis demonstrates high correlation between the moments of kinetic phase transition of the first kind and the concentration of surface-active substances in chemically active materials.

The problems discussed in the current issue enrich both statistical and operations research theoretical modeling and their practical applications in a wide variety of problems of modern science and businesses.

Dr. Alexander Topchishvili

Guest Editor for MASA Issue \#3, Volume 6, 2011 Marburg, Germany
Dr. Stan Lipovetsky

MASA Co-Editor-in-Chief

Minneapolis, USA 